\title{
Compromiso, motivación y persistencia de participantes en xMOOC
}

\section{Engagement, motivation and persistence of XMOOC participants}

\author{
Brenda Edith Guajardo Leal \\ Centro de Estudios para la Enseñanza y Aprendizaje del Derecho. Monterrey, México \\ brendagl@ceead.org.mx \\ Katherina Edith Gallardo Córdova \\ Tecnológico de Monterrey. Monterrey, México \\ katherina.gallardo@tec.mx
}

\begin{abstract}
Resumen
Los cursos masivos y abiertos en línea (MOOC, por sus siglas en inglés Massive, Open Online Courses) se presentan como una modalidad educativa innovadora, económica y de fácil acceso, que ofrece la oportunidad de democratizar la educación en nuestro tiempo. Si bien el interés en estos cursos está en aumento, la tasa de participación de los estudiantes disminuye significativamente a medida que avanzan en las actividades, por lo que la eficiencia terminal suele ser baja. Este estudio cualitativo con diseño de caso múltiple estudió el compromiso, la motivación y la persistencia de los participantes de xMOOC a través del análisis de voces detalladas y descripción profunda de las perspectivas de los participantes, para comprender mejor las formas en las participan de esta modalidad educativa. Los hallazgos revelaron diferencias entre los grupos de alto, medio y bajo rendimiento en las distintas dimensiones del compromiso y persistencia, así como semejanzas en las necesidades psicológicas básicas de competencia, autonomía y relación. Aunque la muestra fue pequeña, fue posible hacer recomendaciones para la toma de medidas específicas con respecto al diseño, la política, la práctica, la teoría y la investigación posterior en cursos en línea masivos y abiertos.
\end{abstract}

Palabras clave: MOOC, compromiso, motivación, persistencia.

\begin{abstract}
Massive Open Online Courses (MOOC) are presented as an innovative, affordable and easily accessible educational modality, offering the opportunity to democratize education in our time. While interest in these courses is on the rise, the participation rate of students decreases significantly as they progress through activities, so terminal efficiency is often low. This qualitative study with multiple case design studied the engagement, motivation and persistence of xMOOC participants through detailed voice analysis and in-depth description of participants' perspectives, to better understand the ways in which they participate in this educational modality. The findings revealed differences between the high, medium and low performance groups in the different dimensions of engagement and persistence, as well as similarities in the basic psychological needs of competence, autonomy and relationship. Although the sample was considered small, it was possible to make recommendations for specific actions regarding design, policy, practice, theory, and subsequent research in massive open online courses.
\end{abstract}

Keywords: MOOC, engagement, motivation, persistence.

RED. Revista de Educación a Distancia. Núm. 66, Vol. 21. Artíc. 10, 30-Abril-2021

DOI: http://dx.doi.org/10.6018/red. 440241 


\section{Introducción}

En el contexto actual, México enfrenta retos y compromisos de gran escala a nivel nacional e internacional relacionados con el sector energético y el desarrollo sostenible. Al reconocer que el gobierno requiere de aliados estratégicos para hacer frente a situaciones tales como la reducción de los impactos medioambientales, surge el proyecto Laboratorio binacional para la gestión inteligente de la sostenibilidad energética y la formación tecnológica. Uno de los objetivos del Laboratorio es la formación de talento especializado en el sector eléctrico, por lo que un conjunto de 12 cursos basados en tecnología XMOOC fueron desarrollados para cubrir las necesidades de capacitación. En contraste con los MOOC tradicionales, los xMOOC siguen un modelo de enseñanza centrado en la transmisión de información con entrega de contenido de alta calidad, evaluación marcada por computadora principalmente para propósitos de retroalimentación de los estudiantes, y automatización de todas las transacciones clave entre los participantes y la plataforma de aprendizaje.

A pesar de que las tasas de finalización de los 12 cursos desarrollados por el Laboratorio binacional no son tan bajas como en la mayoría de los xMOOC ofrecidos en plataformas populares como Coursera o Udacity, las cuales son menores al $10 \%$ (Halawa, Greene, y Mitchell, 2014; Joksimović, et al., 2018; Jordan, 2014), éstas siguen generando preocupación, ya que la eficiencia terminal no supera el 15\% (Guajardo, Valenzuela y Scott, 2019). Las estadísticas de baja finalización son obstáculos que debilitan la sostenibilidad, eficiencia y eficacia de los xMOOC que se ofrecen en el proyecto, ya que la baja participación genera inquietud sobre la capacidad de los cursos de lograr el efecto que se desea en el alumno cuando no asume un papel activo en sus propios procesos de aprendizaje (Joksimović, et al., 2018).

Frente a estos retos, el estudio del engagement o compromiso del estudiante ha tomado relevancia como marco teórico principal para comprender el comportamiento de los estudiantes, para entender las posibles causas del abandono o permanencia escolar, así como para tomar mejores decisiones en el diseño de intervenciones, ya que un conjunto amplio de literatura ha establecido correlaciones sólidas entre el compromiso y los resultados positivos del éxito y desarrollo de los estudiantes (Ferguson y Clow, 2015; Joksimović et al., 2018; Xiong, Li, Kornhaber, Suen, Pursel, y Goins, 2015). El objetivo de este estudio cualitativo de caso múltiple es conocer a profundidad las perspectivas de participantes de xMOOC que se forman en sostenibilidad energética con respecto a su compromiso, motivación y persistencia, para conocer las razones por las cuales completaron o no completaron el curso.

\section{Fundamentos teóricos}

En este apartado se describen algunos fundamentos teóricos y conceptuales que contribuyen al entendimiento de los constructos compromiso, motivación y persistencia. Además de la explicación de los constructos en sí mismos, se explica cómo se asocian entre ellos, y se rescatan algunos esfuerzos investigativos en torno al aprendizaje por medio de MOOC.

\subsection{Compromiso del estudiante en el marco de los MOOC}

Compromiso, motivación y persistencia del participante en xMOOC. Brenda E. Guajardo-Leal,

Katherina E. Gallardo Córdova.

Página 2 de 19 
Los estudios sobre el compromiso del estudiante en los MOOC son recientes. Autores como Anderson, Huttenlocher, Kleinberg y Leskovec (2014) y Kizilcec, PérezSanagustín y Maldonado (2016) argumentan que se comprende poco sobre las formas en las que los estudiantes participan y se involucran en este tipo de ambientes de aprendizaje. Por ello, en los últimos años se han desarrollado propuestas de modelos conceptuales para ayudar a comprender el comportamiento de los participantes, así como mapeos y revisiones sistemáticas de literatura con el mismo fin.

Entre las propuestas conceptuales actuales, destaca la investigación de Redmond, Heffernan, Abawi, Brown y Henderson (2018), quienes desarrollaron un modelo de compromiso en MOOC que comprende cinco dimensiones: conductual, emocional, cognitiva, colaborativa y social. En la dimensión conductual los estudiantes demuestran una conducta y actitud positiva hacia el aprendizaje, participación activa en las actividades, y autorregulación de su aprendizaje; además, los estudiantes apoyan a sus compañeros a seguir procedimientos, a participar activamente en el proceso de aprendizaje, y a reducir conductas disruptivas (Fredricks, Blumenfeld, y Paris, 2004). La dimensión emocional se refiere a las reacciones emocionales y actitudinales de los estudiantes frente al aprendizaje, la disciplina, o hacia las tareas que se espera que los estudiantes realicen. En la dimensión cognitiva los estudiantes se involucran con su propio proceso de aprendizaje, a menudo este componente del compromiso se relaciona con los conceptos de meta-cognición, autorregulación, y aprendizaje estratégico (Pintrich y De Groot, 1990; Redmond et al., 2018; Zimmerman, 2008). La dimensión colaborativa está relacionada con el desarrollo de diferentes redes que apoyan el aprendizaje, incluida la colaboración con compañeros, instructores, la industria y la institución educativa. Por último, la dimensión social es la forma en la que los estudiantes crean relaciones intencionadas con los demás, tanto en actividades académicas como no académicas. Según Krause (2005), "las oportunidades para el compromiso social son tan importantes como las actividades intelectuales" (p. 9).

Por su parte, Joksimović et al. (2018) realizaron una revisión sistemática de literatura sobre los enfoques para modelar el aprendizaje en MOOC, y ofrecieron un análisis de los constructos relacionados con el aprendizaje utilizados en la predicción y medición del compromiso de los estudiantes y de los resultados de aprendizaje. Entre sus resultados discutieron la falta de marcos sólidos para explicar el aprendizaje en un entorno abierto en línea, por lo que presentaron un marco basado en un modelo bien establecido de compromiso estudiantil de Reschly y Christenson (2012). Desde el marco derivado de la investigación de Joksimović et al. (2018) se propuso la asociación entre factores contextuales (es decir, demografía, características del aula y necesidades individuales), compromiso del estudiante (métricas de compromiso académico, conductual, cognitivo y afectivo) y resultados de aprendizaje (académico, social, y afectivo) con el objetivo de guiar la investigación futura.

En otro estudio, Guajardo-Leal, Navarro-Corona y Valenzuela (2019) mapearon sistemáticamente la literatura existente sobre el constructo compromiso en cursos en línea masivos y abiertos en el período de 2015 al 2018. Entre sus resultados identificaron que existía un aumento considerable de artículos publicados que asocian el compromiso académico y los MOOC, principalmente de Estados Unidos, Australia y Reino Unido. Destacaron que había una tendencia hacia los métodos cualitativos, con un enfoque exploratorio, aunque también encontraron diversos estudios correlacionales. Otro de sus hallazgos fue encontrar que el estudio de los patrones de participación, el

Compromiso, motivación y persistencia del participante en xMOOC. Brenda E. Guajardo-Leal, Katherina E. Gallardo Córdova. 
diseño instruccional, la motivación y la persistencia, aparecieron como temas asociados al campo del compromiso estudiantil.

\subsection{Constructos asociados con el compromiso del estudiante}

Diversas investigaciones han estudiado la relación del compromiso del estudiante con otros constructos psicológicos, entre ellos el de motivación y persistencia (GuajardoLeal et al., 2019; Xiong, Kornhaber, Suen, Pursel y Goins, 2015; Wen, Yanh y Rosé, 2014). La motivación es el proceso interno del ser humano que le brinda la energía para poder iniciar, dirigir y mantener un comportamiento orientado a satisfacer una necesidad (Bandura, 2006); el compromiso, en cambio, es considerado la manifestación de la motivación (Reeve, 2012), es decir, es la respuesta física, cognitiva y emocional dada frente a una necesidad. La persistencia se define como "la continuación voluntaria de una acción dirigida a un objetivo a pesar de los obstáculos, las dificultades o el desaliento" (Peterson y Seligman, 2004, p. 229); es entonces la continuación perseverante y voluntaria del compromiso.

Específicamente sobre la motivación, actualmente coexisten teorías que han identificado y estudiado sus diversas fuentes. La teoría de la auto-determinación de Deci y Ryan (1985; 2010) (SDT, por sus siglas en inglés, Self-determination Theory) es sin duda una de las más reconocidas. La SDT asume que todos los estudiantes poseen tendencias de crecimiento inherentes o innatas que proporcionan una base para su participación en el aula y su funcionamiento escolar efectivo (Deci y Ryan, 2010). Dentro de la SDT, la teoría de las necesidades básicas busca explicar cómo las necesidades de autonomía, competencia y relación afectan las acciones de las personas; estas necesidades universales son los nutrientes psicológicos esenciales a lo largo de la vida para el "crecimiento psicológico, la integridad y el bienestar continuos" (Deci y Ryan, 2000, p. 229). La teoría de la auto-determinación ha demostrado que cuando se satisfacen estas tres necesidades se predicen una serie de resultados funcionales positivos en términos de motivación, autorregulación, aprendizaje, organización, vitalidad y bienestar continuos (Appleton, Christenson, y Furlong, 2008; Martin, Kelly, y Terry, 2018; Reeve, 2012).

Autores como Appleton et al. (2008) definen a la necesidad de autonomía como la elección que se tiene en la iniciación, mantenimiento y regulación de la actividad y la experiencia de conexión entre las acciones, las metas, y los valores personales. La competencia se relaciona con una necesidad innata de aprender y dominar nuevas habilidades y capacidades para ser efectivo en actividades que son importantes para el individuo (White, 1959). La relación es la necesidad de establecer vínculos emocionales cercanos con los demás para sentirse conectados (Reeve, 2012); refleja el deseo de estar emocionalmente enlazado e interpersonalmente involucrado en relaciones cálidas, afectuosas y receptivas (Deci y Ryan, 1991).

En lo referente a la persistencia, Tinto (2017) sugiere dos aspectos relacionados con las teorías de motivación: la autoeficacia percibida y el valor percibido del currículo. La autoeficacia se refiere a las creencias de los estudiantes sobre sus propias capacidades para organizar y ejecutar cursos de acción (Bandura, 1977). Los individuos con un fuerte sentido de autoeficacia: a) ven los problemas desafiantes como tareas que deben dominarse; b) desarrollan un interés más profundo en las actividades en las que participan; c) forman un mayor sentido de compromiso con sus intereses y actividades; y d) se recuperan rápidamente de las adversidades y decepciones (Bandura, 1995); este último punto se relaciona con el concepto de resiliencia, que es la capacidad que tiene

Compromiso, motivación y persistencia del participante en xMOOC. Brenda E. Guajardo-Leal, Katherina E. Gallardo Córdova. 
una persona de recuperarse frente a la adversidad para seguir proyectando hacia el futuro, y con la teoría del flujo de Csikszentmihályi $(1996 ; 2008)$ la cual indica que las personas experimentan un estado psicológico óptimo cuando participan en una actividad que es a la vez un desafío apropiado para su nivel de habilidad. Según los reportes académicos, las personas con alta autoeficacia tenderán a involucrarse más fácilmente en una tarea, harán un mayor esfuerzo y persistirán más tiempo en completarla, incluso cuando encuentren dificultades (Chemers, Hu, y Garcia, 2001). El valor percibido hacia el currículum se refiere a las percepciones de los estudiantes sobre el valor de lo que se les pide aprender (Tinto, 2017); para persistir, los estudiantes deben percibir que el material que se debe aprender es de suficiente calidad y relevancia (Tessema, Ready, y $\mathrm{Yu}, 2012)$.

La relación entre el compromiso estudiantil, la motivación y la persistencia en MOOC ha sido estudiada previamente en estudios cuantitativos. Por ejemplo, en su estudio, Xiong et al., (2015) por medio de un modelo de ecuaciones estructurales encontraron que la motivación predice significativamente la participación de los estudiantes en el curso, y que además el compromiso es un fuerte predictor de retención. Sus hallazgos sugirieron utilizar el monitoreo y seguimiento de estudiantes de forma personalizada para mejorar la retención en este tipo de ambientes de aprendizaje. En otro estudio, Wen et al., (2014), por medio de un análisis de supervivencia, encontraron que la motivación del estudiante, medida por porcentaje de participación en publicaciones y la participación cognitiva (p. ej. participación en foros), fueron predictores significativos de deserción; los autores concluyeron que las interacciones en redes sociales que regularmente tienen lugar en foros de discusión y publicaciones, pueden influir en la motivación del estudiante, y por lo tanto, en su persistencia en el curso.

Por su parte, Yuan y Powell (2013) realizaron una revisión de la literatura que se centró en la amplia información de los MOOC a través de blogs académicos, comunicados de prensa, así como informes y artículos de investigación, para identificar los debates actuales sobre la oferta de nuevos cursos, el impacto de los cambios en la financiación y las implicaciones para una mayor apertura en la educación superior. Entre sus resultados destacaron que la motivación, específicamente factores internos como la curiosidad y el disfrute, y externos como el beneficio económico a futuro, contribuyen significativamente a las tasas de deserción en MOOC.

Es posible que, con un estudio profundo cualitativo sobre el compromiso y sus dos constructos asociados, la motivación y la persistencia, se logre una mejor comprensión de las formas en las que los estudiantes participan en los xMOOC. Profundizar en el estudio de factores internos o endógenos al participante, permitirá ampliar los conocimientos existentes sobre los factores relacionados con su rendimiento y las razones por las cuales culminan o desisten en este tipo de modalidad educativa.

\section{Metodología}

Estudio de corte cualitativo con el objetivo de conocer las perspectivas de los participantes de xMOOC que se forman en sostenibilidad energética con respecto a su compromiso, motivación y persistencia, para comprender cuáles son las percepciones de los participantes de $\mathrm{xMOOC}$ con respecto a las razones por las cuales permanecieron $\mathrm{o}$ desertaron en el curso.

Se utilizó como aproximación metodológica un diseño de estudio de caso múltiple (Stake, 1995; Yin, 2003). La selección del diseño de este estudio partió del supuesto 
filosófico constructivista que valora las perspectivas múltiples y la descripción profunda de los resultados (Creswell y Plano-Clark, 2018).

\subsection{Selección de casos}

La unidad de análisis fueron participantes de 12 xMOOC desarrollados por el Programa Binacional en su última impartición del 2019. La muestra para este estudio estuvo conformada por 10 estudiantes que aceptaron participar en una entrevista semiestructurada virtual de manera voluntaria y que se escogieron porque reportaron intención de terminar el xMOOC al que estaban inscritos. Se utilizaron procedimientos para una muestra propositiva para entender a profundidad el fenómeno (Creswell, 2012). La estrategia para la selección fue el muestreo de máxima variación, esperando que los individuos seleccionados tuvieran diferentes perspectivas del fenómeno bajo estudio. Se eligió como criterio de máxima variación el rendimiento del participante, considerando dentro de la muestra cualitativa a cuatro estudiantes que terminaron con rendimiento alto, dos que terminaron con rendimiento medio, y cuatro que no terminaron alguno de los cursos, con la intención de llevar a cabo una comparación cualitativa profunda de cómo se comportaron estas tres categorías de análisis.

De la muestra seleccionada, el $80 \%$ fueron hombres y $20 \%$ mujeres con una edad promedio de 35.2 años. Reportaron ser originarios de distintos países: (5) México, (2) Bolivia, (1) República dominicana, (1) Argentina, y (1) Colombia. El 50\% de los entrevistados mencionaron no tener experiencia laboral alguna en el área de energía o sostenibilidad energética, mientras el resto reportaron tener entre 1 y 28 años de experiencia. El 80\% de los entrevistados terminó el curso con el $60 \%$ de las actividades realizadas, y del total, el $50 \%$ se certificó. El perfil de los participantes fue variado: estudiantes (3), empleados (4), estudiante/empleado (1), empresario (1) y desempleado (1).

\subsection{Instrumentación}

Se diseñaron dos instrumentos: una lista de cotejo y un guion de entrevista semiestructurada. La lista de cotejo incluyó un repertorio de 16 estrategias de aprendizaje reportadas en la literatura sobre el compromiso estudiantil (Pintrich y De Groot, 1990; Redmond et al., 2018; Zimmerman, 2008), con ella se analizaron un total de 17 fotografías de notas o apuntes realizados por los participantes durante el curso. La entrevista recuperó las percepciones de los participantes con respecto a su motivación, compromiso y persistencia; constó de 18 preguntas divididas en tres secciones. En la primera sección se abordaron las instrucciones, objetivo del estudio y se hicieron preguntas demográficas. En la segunda sección, se abordaron preguntas relacionadas con la motivación, específicamente sobre las necesidades de competencia, autonomía y relación, preguntas relacionadas con el compromiso que abarcó emociones, estrategias cognitivas, conducta, disposición para la colaboración y para las relaciones sociales durante el curso, y además, se abordaron preguntas encaminadas a conocer la voluntad, autoeficacia, resiliencia y el valor que los participantes le tienen a las actividades del curso. Por último, en la tercera sección se hicieron preguntas de cierre relacionadas con la participación y proyección hacia el futuro del estudiante en este tipo de cursos. El contenido del protocolo de la entrevista fue probado por un participante seleccionado propositivamente. Como resultado, algunas preguntas se modificaron ligeramente y se desarrollaron preguntas adicionales.

Compromiso, motivación y persistencia del participante en xMOOC. Brenda E. Guajardo-Leal,

Katherina E. Gallardo Córdova.

Página 6 de 19 


\subsection{Procedimientos}

Los pasos para el análisis ocurrieron de forma simultánea e iterativa (Creswell y Guetterman, 2019); estos fueron: preparar las bases de datos para el análisis, explorar los datos, analizar los datos, representar el análisis, interpretar el análisis y validar el estudio. Para analizar las fotografías se contrastó la lista de cotejo con las estrategias de aprendizaje utilizadas en las notas de los participantes. Para las entrevistas se utilizó un análisis inductivo que va de lo particular (trascripciones) a lo general (códigos y categorías emergentes). Cada entrevista fue audio-grabada y transcrita literalmente. El análisis se llevó a cabo en dos niveles: en cada caso y entre los casos (Stake, 1995, Yin, 2003). Los pasos en el análisis de las entrevistas incluyeron: exploración de los datos, codificación por segmentación y etiquetado de texto, verificación de códigos a través de la verificación de acuerdo entre ellos, agrupación de códigos en temas, conexión e interrelación de categorías, descripción de los temas, construcción de la narrativa de estudio de caso con las descripciones de los temas, y un análisis temático de casos cruzados. La credibilidad de los hallazgos se aseguró mediante la triangulación de diferentes fuentes de información, verificación de miembros, descripciones completas y exhaustivas de los casos, y revisión y resolución de evidencia disconforme (Creswell, 1998; Stake, 1995).

\section{Resultados}

Del análisis de cada caso y a través de los 10 casos emergieron tres temas: (1) Motivación para entrar al xMOOC, (2) Compromiso con el curso, (3) Persistencia. A continuación, los 10 casos se agrupan según su rendimiento: alto, medio y bajo, y dentro de cada uno de ellos se describen los temas emergentes.

\subsection{Percepciones de participantes sobre su motivación, compromiso y persistencia: explicaciones detrás de su rendimiento.}

\subsubsection{Rendimiento alto}

Roberto, Esteban, Jorge y Cecilia conforman el grupo de casos de rendimiento alto con una calificación final mayor a 0.8. Ellos se inscribieron al curso para reforzar sus conocimientos prácticos o teóricos o porque consideraban que la energía era un área endeble en su formación. Otro aspecto que los motivó a inscribirse fue la modalidad en línea, la cual les brindó flexibilidad en horarios y completa accesibilidad al material. Los participantes también indicaron haberse inscrito al curso por el idioma de impartición y la temática de vanguardia, la inversión monetaria, el crecimiento laboral, y el amor por aprender.

Sobre el compromiso personal, los participantes de rendimiento alto planearon y organizaron su rutina de estudio, incluyendo una calendarización diaria o semanal, además de organización de los materiales de estudio; resaltaron la importancia de involucrarse con "orden, tiempo y amor al conocimiento". Los participantes en este grupo demostraron estar emocionados frente algunas de las actividades diseñadas, por ejemplo, aquellas que requerían aplicar el conocimiento a una situación de su vida diaria: "el reto, ese me encantó". Utilizaron estrategias cognitivas de repaso cuando volvían a realizar las actividades o evaluaciones al momento de identificar los errores cometidos, o cuando se les hacía difícil algún contenido, y realizaron re-visitas a videos o lecturas. También utilizaron estrategias de elaboración al tomar notas no literales, digitales o en su libreta, al elaborar resúmenes, y al tomar fotos de pantalla para asegurar el resguardo de contenidos importantes, y estrategias de organización al realizar dibujos, gráficos o tablas. Asimismo, utilizaron estrategias de búsqueda de 
información en otras fuentes fuera del curso (e.g. internet) para complementar lo aprendido.

En cuanto al compromiso contextual, sólo un participante de este grupo colaboró con otros compañeros en las actividades que se solicitaron en el curso (e.g. coevaluaciones). Aunque la colaboración con otros enriqueció su experiencia, ya que aprendió diferentes maneras de resolver un problema: "yo resolví por teorema de Pitágoras para el cálculo de energía reactiva, y ellos utilizaron tangente inversa, entonces [identifiqué] diferentes maneras de resolver un problema", no participó en los foros de discusión, ya que él, como el resto de los participantes de este grupo, consideró que la forma en la que están organizados los foros de discusión no es la óptima, pues entre los miles de participantes sólo algunos comentarios obtienen respuesta y continuidad, por lo que la gran mayoría de las dudas quedan sin resolver. El resto de los participantes prefirieron no relacionarse con otros compañeros porque notaron cierta distancia en el canal diseñado para vincularse o comunicarse con otros. No obstante, los participantes también mencionaron que les hubiera gustado pertenecer a alguna red social de apoyo para afrontar dificultades. Sobre la persistencia, todos los participantes de rendimiento alto se propusieron como meta al inicio del curso terminarlo y obtener el certificado, creían en sus capacidades para lograrlo. Frente a la adversidad, buscaron en otras fuentes o pidieron ayuda fuera del curso.

\subsubsection{Rendimiento medio}

David y Salvador conforman el grupo de rendimiento medio con calificaciones mayores a 0.6 pero menores a 0.8 . La motivación de Salvador para entrar al curso fue reforzar conocimientos para tener un trabajo, en cambio, la motivación de David fue adquirir nuevo conocimiento que complementara sus estudios universitarios. Ambos dieron especial importancia a la flexibilidad que ofrecen los cursos en cuanto al lugar, tiempo y duración, y a la disponibilidad del material, el cual es de consulta a libre demanda. Ninguno se interesó en relacionarse con otros compañeros.

Con respecto a las dimensiones del compromiso, ambos organizaron su rutina de estudio y establecieron metas. Reportaron haber utilizado estrategias de repaso, entre ellas la repetición de ejercicios, el subrayado y la toma de fotografías: "una vez hechas las notas yo trataba de subrayar y luego hacía más notas sobre las notas"; estrategias de elaboración, al hacer resúmenes de ideas principales y al tomar notas no literales; y estrategias de organización, cuando realizaron gráficos o tablas con la información del curso. No buscaron en otras fuentes fuera del curso, ni pidieron ayuda a otras personas, ya que el contenido les pareció poco retador. Sólo uno de ellos expresó emoción y pasión por el tema de estudio, especialmente frente al diseño de actividades aplicadas: "me sentí muy involucrado y me encantaron esas actividades que puedes llevar a tu vida laboral o a tu propia casa". Sobre el compromiso contextual, su intención al ingresar en el curso no fue para conocer a más gente interesada en el área. Uno de ellos participó en dos ocasiones en el foro sin involucrarse en discusiones. Ambos resaltaron que pertenecer a un grupo de red social por medio de WhatsApp u otra aplicación pudiera haber sido útil para despejar sus dudas de manera más efectiva. En cuanto a la persistencia, ambos ingresaron al curso con la intención de terminarlo y certificarse, mencionaron que creían en su capacidad para completarlo. Ninguno de ellos encontró que el contenido fuera retador.

Compromiso, motivación y persistencia del participante en xMOOC. Brenda E. Guajardo-Leal, 


\subsubsection{Rendimiento bajo}

Cesar, José, Marcos y Martha integran el grupo de bajo rendimiento con calificaciones finales menores a 0.6. Marcos y Cesar ingresaron al MOOC con intención de realizar sólo algunas de las actividades, es decir, sin intención de culminarlo. Aunque su meta fue leer y aprender el contenido del curso que les interesaba, se identificaron como participantes que deseaban terminarlo, ya que para ellos cumplir con esa meta significaba terminar con éxito. José y Martha mencionaron al inicio del curso su interés por realizar todas las actividades, sin lograrlo.

En lo referente a su motivación, los casos son muy diversos. Cesar ingresó al curso para repasar y ampliar su conocimiento, no buscó certificarse. José se inscribió al curso para apoyar su formación profesional y ampliar su conocimiento técnico, pero esta necesidad se no se satisfizo; también afirmó haberse inscrito al curso por su interés de laborar en el extranjero. Marcos afirmó que el campo de la energía no era su área de estudio, tomó el curso para comenzar a aprender sobre el tema; no buscó certificarse. En su punto de vista, satisfizo esa necesidad de conocimiento debido al diseño del curso y a la calidad de los recursos. Martha se interesó en cursar el MOOC para tener un panorama del área de energía. Esta necesidad no se satisfizo ya que no contaba con los conocimientos básicos en materia de energía y fue difícil para ella seguir el curso. Ninguno ingresó al curso con la intención de relacionarse con otros. Todos los casos mencionaron haberse inscrito a estos cursos por la facilidad de llevarlo por su cuenta, sin un horario ni lugar fijo de estudio.

Sobre su compromiso personal y contextual con el curso, ninguno de los participantes en este grupo especificó una rutina para llevar a cabo las actividades del curso, no siguieron el calendario del curso ni uno propio, mas bien, fueron a su ritmo leyendo y realizando las actividades. En cuanto a las estrategias cognitivas, los integrantes de este grupo sólo reportaron haber utilizado estrategias de repaso, entre ellas la repetición o práctica de ejercicios, la toma de notas literales, y la toma de fotografías. Sólo un integrante de este grupo participó en una actividad colaborativa, pero su experiencia no fue placentera, comentó que la evaluación que le brindaron no fue justa y que el diseño del curso no permitía oportunidad de réplica, por lo que decidió no participar en el resto de las actividades colaborativas. Los demás participantes resaltaron que no había interés por parte de sus pares en las actividades colaborativas porque no identificaron un canal de comunicación eficiente. Sobre la participación en foros, éstos les parecieron difíciles de dar seguimiento y no los motivaron a participar por la forma en la que están diseñados para las masas. Los integrantes de este grupo concordaron en que les hubiera gustado involucrarse en redes sociales para intercambiar información.

En lo que respecta a la persistencia, dos integrantes ingresaron al curso con la intención de terminarlo, aunque su definición de "terminación" estaba más bien relacionada con obtener la información que le interesaba del curso sin necesariamente realizar todos los ejercicios; el resto de los participantes buscaban terminar el curso y certificarse. Todos creían en sus propias capacidades para lograr sus metas. Los comentarios sobre el contenido y la estructura del curso fueron en su mayoría negativos, videos "un tanto lentos", recursos "largos y técnicos", falta de explicaciones conceptuales, o enfoque estrecho. Para dos participantes el contenido del curso les pareció bastante básico, no reportaron haberse encontrado con situaciones difíciles o adversas; el curso no significó un reto para ellos, por lo que no lograron motivarse a realizar las actividades completas. Para dos de los participantes la temática del curso no era prioritaria para su campo de

Compromiso, motivación y persistencia del participante en xMOOC. Brenda E. Guajardo-Leal, 
estudio, y además, consideraron que el contenido era muy retador y no se empataba con sus conocimientos básicos en el área.

\subsection{Razones que explican la permanencia o deserción en el xMOOC}

Para conocer las explicaciones detrás de su retención o deserción, se llevó a cabo un análisis de casos cruzados. De los tres temas que emergieron del primer análisis: compromiso, motivación y persistencia, a pesar de ser comunes para todos los participantes, estos difirieron en número y semejanza de sub-temas y categorías que los conformaron (ver Tabla 1).

Tabla 1

Temas, sub-temas y categorías a través de los casos

\begin{tabular}{|c|c|c|c|}
\hline $\begin{array}{l}\text { Temas y sub- } \\
\text { temas }\end{array}$ & Rendimiento alto & Rendimiento medio & Rendimiento bajo \\
\hline \multicolumn{4}{|l|}{ Compromiso } \\
\hline \multirow{8}{*}{$\begin{array}{l}\text { Compromiso } \\
\text { personal } \\
\text { (cognitivo, } \\
\text { emocional, } \\
\text { conductual) }\end{array}$} & $\begin{array}{l}\text { Planeación y } \\
\text { organización de rutina } \\
\text { (uso de calendario) }\end{array}$ & $\begin{array}{l}\text { Planeación y } \\
\text { organización de rutina } \\
\text { (uso de calendario) }\end{array}$ & \\
\hline & $\begin{array}{l}\text { Estrategias de repaso } \\
\text { (repetición, re-visitas, } \\
\text { toma de notas }\end{array}$ & $\begin{array}{l}\text { Estrategias de repaso } \\
\text { (repetición, re-visitas, } \\
\text { toma de notas }\end{array}$ & $\begin{array}{l}\text { Estrategias de repaso } \\
\text { (repetición, re-visitas, } \\
\text { toma de notas }\end{array}$ \\
\hline & $\begin{array}{l}\text { literales, fotografías, } \\
\text { subrayar) }\end{array}$ & $\begin{array}{l}\text { literales, fotografías, } \\
\text { subrayar) }\end{array}$ & $\begin{array}{l}\text { literales, fotografías, } \\
\text { subrayar) }\end{array}$ \\
\hline & $\begin{array}{l}\text { Estrategias de } \\
\text { elaboración (toma de } \\
\text { notas no literales, } \\
\text { resumir) }\end{array}$ & $\begin{array}{l}\text { Estrategias de } \\
\text { elaboración (toma de } \\
\text { notas no literales, } \\
\text { resumir) }\end{array}$ & \\
\hline & $\begin{array}{l}\text { Estrategias de } \\
\text { organización (tablas, } \\
\text { gráficos, mapas) }\end{array}$ & $\begin{array}{l}\text { Estrategias de } \\
\text { organización (tablas, } \\
\text { gráficos, mapas) }\end{array}$ & \\
\hline & $\begin{array}{l}\text { Búsqueda en otras } \\
\text { fuentes (internet) }\end{array}$ & & \\
\hline & $\begin{array}{l}\text { Pasión / Amor por el } \\
\text { tema }\end{array}$ & $\begin{array}{l}\text { Pasión / Amor por el } \\
\text { tema }\end{array}$ & \\
\hline & $\begin{array}{l}\text { Búsqueda de ayuda / } \\
\text { asesoría }\end{array}$ & & $\begin{array}{l}\text { Búsqueda de ayuda / } \\
\text { asesoría }\end{array}$ \\
\hline \multirow{3}{*}{$\begin{array}{c}\text { Compromiso } \\
\text { contextual } \\
\text { (colaborativo y } \\
\text { social) }\end{array}$} & Evaluación entre pares & & $\begin{array}{l}\text { Evaluación entre } \\
\text { pares sensación de } \\
\text { injusticia }\end{array}$ \\
\hline & $\begin{array}{l}\text { Pertenecer a un grupo } \\
\text { de apoyo o red social } \\
\text { Foros muy amplios }\end{array}$ & $\begin{array}{l}\text { Pertenecer a un grupo } \\
\text { de apoyo o red social } \\
\text { Foros muy amplios }\end{array}$ & $\begin{array}{l}\text { Pertenecer a un grupo } \\
\text { de apoyo o red social } \\
\text { Foros muy amplios }\end{array}$ \\
\hline & $\begin{array}{l}\text { Sin espacios para } \\
\text { interacción con redes } \\
\text { sociales }\end{array}$ & $\begin{array}{l}\text { Sin espacios para } \\
\text { interacción con redes } \\
\text { sociales }\end{array}$ & $\begin{array}{l}\text { Sin espacios para } \\
\text { interacción con redes } \\
\text { sociales }\end{array}$ \\
\hline
\end{tabular}


RED. Revista de Educación a Distancia. Núm. 66, Vol. 21. Artíc. 10, 30-Abril -2021

DOI: https://doi.org/10.6018/red.440241

\begin{tabular}{|c|c|c|c|}
\hline $\begin{array}{l}\text { Temas y sub- } \\
\text { temas }\end{array}$ & Rendimiento alto & Rendimiento medio & Rendimiento bajo \\
\hline \multicolumn{4}{|l|}{ Motivación } \\
\hline $\begin{array}{l}\text { Necesidad de } \\
\text { conocimiento }\end{array}$ & $\begin{array}{l}\text { Reforzar conocimiento } \\
\text { técnico } \\
\text { Adquirir nuevo } \\
\text { conocimiento }\end{array}$ & $\begin{array}{l}\text { Reforzar } \\
\text { conocimiento técnico } \\
\text { Adquirir nuevo } \\
\text { conocimiento }\end{array}$ & $\begin{array}{l}\text { Reforzar } \\
\text { conocimiento técnico } \\
\text { Adquirir nuevo } \\
\text { conocimiento } \\
\text { Falta de } \\
\text { conocimientos } \\
\text { técnicos } \\
\text { Falta de aplicación } \\
\text { de conocimiento } \\
\text { Temas sin prioridad }\end{array}$ \\
\hline \multirow{4}{*}{$\begin{array}{l}\text { Necesidad de } \\
\text { autonomía }\end{array}$} & $\begin{array}{l}\text { Autoevaluación del } \\
\text { propio avance }\end{array}$ & & \multirow{4}{*}{$\begin{array}{l}\text { Autoevaluación del } \\
\text { propio avance } \\
\text { Flexibilidad de } \\
\text { tiempo (horarios de } \\
\text { estudio) } \\
\text { Accesibilidad al } \\
\text { material } \\
\text { Flexibilidad de } \\
\text { espacio }\end{array}$} \\
\hline & $\begin{array}{l}\text { Flexibilidad de tiempo } \\
\text { (horarios de estudio) }\end{array}$ & $\begin{array}{l}\text { Flexibilidad de tiempo } \\
\text { (horarios de estudio) }\end{array}$ & \\
\hline & $\begin{array}{l}\text { Accesibilidad al } \\
\text { material }\end{array}$ & $\begin{array}{l}\text { Accesibilidad al } \\
\text { material }\end{array}$ & \\
\hline & $\begin{array}{l}\text { Flexibilidad de } \\
\text { espacio }\end{array}$ & $\begin{array}{l}\text { Flexibilidad de } \\
\text { espacio }\end{array}$ & \\
\hline $\begin{array}{l}\text { Necesidad de } \\
\text { relación }\end{array}$ & $\begin{array}{l}\text { Sin vínculo } \\
\text { comunicativo con el } \\
\text { profesor } \\
\text { Sin vínculo } \\
\text { comunicativo eficiente } \\
\text { con los participantes }\end{array}$ & $\begin{array}{l}\text { Sin vínculo } \\
\text { comunicativo con el } \\
\text { profesor } \\
\text { Sin vínculo } \\
\text { comunicativo eficiente } \\
\text { con los participantes }\end{array}$ & $\begin{array}{l}\text { Sin vínculo } \\
\text { comunicativo con el } \\
\text { profesor } \\
\text { Sin vínculo } \\
\text { comunicativo } \\
\text { eficiente con los } \\
\text { participantes }\end{array}$ \\
\hline \multirow{3}{*}{$\begin{array}{l}\text { Diseño del } \\
\text { curso }\end{array}$} & $\begin{array}{l}\text { Tema de vanguardia } \\
\text { Idioma } \\
\text { Aplicación del } \\
\text { conocimiento } \\
\text { Claridad en el } \\
\text { contenido }\end{array}$ & $\begin{array}{l}\text { Aplicación del } \\
\text { conocimiento }\end{array}$ & \\
\hline & & $\begin{array}{l}\text { Calidad de los } \\
\text { recursos }\end{array}$ & $\begin{array}{l}\text { Calidad de los } \\
\text { recursos } \\
\text { Materiales extensos }\end{array}$ \\
\hline & & & $\begin{array}{l}\text { Falta de contenido } \\
\text { técnico } \\
\text { Videos lentos } \\
\text { Falta de aplicación } \\
\text { del conocimiento } \\
\text { Evaluación injusta } \\
\text { sin derecho a réplica } \\
\text { Contenido local }\end{array}$ \\
\hline
\end{tabular}

Certificación Recuperar dinero 
RED. Revista de Educación a Distancia. Núm. 66, Vol. 21. Artíc. 10, 30-Abril -2021

DOI: https://doi.org/10.6018/red.440241

\begin{tabular}{|c|c|c|c|}
\hline $\begin{array}{c}\text { Temas y sub- } \\
\text { temas }\end{array}$ & Rendimiento alto & Rendimiento medio & Rendimiento bajo \\
\hline & invertido & & \\
\hline & $\begin{array}{l}\text { Mejor trabajo } \\
\text { Mejor sueldo } \\
\text { Subir a red social }\end{array}$ & $\begin{array}{l}\text { Encontrar trabajo } \\
\text { Mejorar sueldo }\end{array}$ & \\
\hline & & Viajar a otro país & $\begin{array}{l}\text { Laborar en el } \\
\text { extranjero }\end{array}$ \\
\hline Red social & $\begin{array}{l}\text { Pertenecer a un grupo } \\
\text { de apoyo o red social }\end{array}$ & & $\begin{array}{l}\text { Pertenecer a un } \\
\text { grupo de apoyo o red } \\
\text { social }\end{array}$ \\
\hline \multicolumn{4}{|l|}{ Persistencia } \\
\hline Voluntad & $\begin{array}{l}\text { Buscan completar el } \\
\text { cuso }\end{array}$ & $\begin{array}{l}\text { Buscan completar el } \\
\text { cuso }\end{array}$ & $\begin{array}{l}\text { Buscan completar el } \\
\text { cuso (solo lo que les } \\
\text { interesa) }\end{array}$ \\
\hline Resiliencia & Contenidos retadores & $\begin{array}{l}\text { Pocos contenidos } \\
\text { retadores }\end{array}$ & $\begin{array}{l}\text { Sin contenidos } \\
\text { retadores } \\
\text { Contenidos } \\
\text { demasiado retadores } \\
\text { para principiantes }\end{array}$ \\
\hline Valor & $\begin{array}{l}\text { Tema de vanguardia } \\
\text { Valor a las actividades } \\
\text { / contenido del curso }\end{array}$ & $\begin{array}{l}\text { Valor a las actividades } \\
\text { / contenido del curso }\end{array}$ & $\begin{array}{l}\text { Tema sin relevancia } \\
\text { Descontento con } \\
\text { materiales del curso } \\
\text { Contenido no abona } \\
\text { a campo de estudio }\end{array}$ \\
\hline Autoeficacia & $\begin{array}{l}\text { Confianza en sí } \\
\text { mismos }\end{array}$ & $\begin{array}{l}\text { Confianza en sí } \\
\text { mismos }\end{array}$ & $\begin{array}{l}\text { Confianza en sí } \\
\text { mismos }\end{array}$ \\
\hline
\end{tabular}

Nota. En itálica se muestran las categorías con connotación negativa.

\section{Discusión y conclusiones}

\subsection{Percepciones sobre compromiso}

El análisis reveló diferencias y semejanzas entre los grupos de alto, medio y bajo rendimiento en las distintas dimensiones del compromiso. Las semejanzas más marcadas fueron en el compromiso contextual: colaborativo y social. Los participantes no estuvieron comprometidos para colaborar con otros; la dimensión colaborativa del modelo de Redmond et al. (2018) no tuvo propósitos académicamente valiosos. Asimismo, los participantes en todos los casos mencionaron haber estado interesados en formar parte de alguna red social como apoyo, con ello se respalda la dimensión social del mismo modelo y se rescata la importancia de las actividades que creen vínculos sociales manifestadas por Krause (2005).

Las diferencias más sobresalientes entre los tres grupos de casos fueron en el compromiso personal: conductual, emocional y cognitivo. Los participantes de rendimiento alto y medio se mostraron interesados por aprender el contenido del curso. En el caso de los participantes con rendimiento bajo, el contenido del curso no fue de relevancia para dos casos, y para el resto, el contenido fue muy difícil o muy fácil de seguir. Al no encontrarle relevancia personal al contenido, los participantes de 
rendimiento bajo no estuvieron comprometidos conductualmente, por lo que no participaron de forma activa de su proceso de aprendizaje, por ejemplo, no buscaron ayuda frente a contenidos difíciles. Estos hallazgos relacionados con la conducta son consistentes con la investigación del compromiso y la persistencia en ambientes presenciales (Fredricks et al., 2004; Tessema, Ready y Yu, 2012; Tinto, 2017) y en línea (Joksimović et al., 2018).

En cuanto a las reacciones emocionales y actitudinales de los participantes, los grupos de rendimiento alto y medio mencionaron tener pasión y emoción por aprender sobre la temática de estudio, materializando su reacción emocional en la aplicación del esfuerzo; por el contrario, los participantes de rendimiento bajo, a pesar de que buscaban aprender los contenidos, hicieron referencia a que la temática no era prioritaria para ellos o a que ya dominaban los contenidos, materializando su reacción emocional en la disminución del esfuerzo. Estos hallazgos son consistentes con el modelo de compromiso de Redmond et al. (2018) y con estudios de psicología cognitiva en ambientes presenciales (Mosher y MacGowan, 1985).

Con respecto al compromiso cognitivo, los participantes en los tres grupos de casos se involucraron con su propio proceso de aprendizaje con diferentes niveles de inmersión, marcados por el uso de estrategias de aprendizaje. Por un lado, todos los participantes reportaron haber utilizado las estrategias de aprendizaje consideradas poco profundas, que incluyen la revisión, el ensayo y la memorización. Las estrategias de este nivel superficial generalmente sirven para incorporar nueva información en la memoria a corto plazo (Zimmerman, 2008). Por otro lado, las estrategias de aprendizaje consideradas de elaboración que ayudan a los estudiantes a almacenar información en la memoria a largo plazo mediante la construcción de conexiones internas entre los elementos para integrar y a conectar nueva información con el conocimiento previo, fueron utilizadas sólo por los estudiantes de rendimiento alto y medio, con un mayor repertorio para los participantes de rendimiento alto. Asimismo, las estrategias de organización que ayudan al aprendiz a seleccionar información relevante y hacer conexiones (elaboración de tablas, figuras, gráficos), también fueron utilizadas por los participantes de rendimiento alto y medio. Por último, la planeación y organización de rutina de estudio fue una de las estrategias de aprendizaje metacognitiva utilizada también por los estudiantes de rendimiento alto y medio, los estudiantes con rendimiento bajo trabajaban cuando tenían el tiempo de hacerlo, sin estructura ni constancia, demostrando así que la planificación de la rutina de estudio es crucial cuando se tiene intención de cumplir con una meta educativa (Zimmerman, 2008). Estos hallazgos en xMOOC hacen eco en las investigaciones que asocian el aprendizaje autoregulatorio y estratégico con el éxito académico o la culminación de una meta educativa (Pintrich y De Groot, 1990; Redmond et al., 2018; Zimmerman, 2008), y hacen reflexionar sobre la importancia de una educación estratégica, no solamente durante la infancia y la juventud, sino también en la edad adulta, que le sirva a las personas permanentemente, y que les permita, a través del uso consciente de estrategias de autorregulación, aprender a aprender. Esta competencia, aprender a aprender, fue fundamental para motivarse, persistir y comprometerse con el curso.

\subsection{Percepciones sobre motivación}

Derivado del análisis, los hallazgos revelaron que los participantes en todos los casos buscaban saciar una necesidad de conocimiento, pero en el caso de los participantes de rendimiento bajo, esa necesidad no se satisfizo. Asimismo, los participantes en todos los 
casos se sintieron atraídos por las características de los MOOC, tales como la flexibilidad en tiempos y lugar para cursarlos, atributos que permitieron mantener los horarios laborales y personales mientras tomaban el curso. Una segunda característica importante fue la flexibilidad de aprender a su propio ritmo con oportunidad de acceder a los materiales en cualquier momento. Estos hallazgos en xMOOC son consistentes con la necesidad psicológica básica de autonomía de teoría de la autodeterminación de Deci y Ryan (1985; 2010). No obstante, ninguno de los participantes en los tres grupos estuvo motivado en relacionarse con otros, aunque algunos de ellos sí lo buscaron, no lograron establecer vínculos comunicativos eficientes. Estos hallazgos nos indican que en este tipo de cursos los participantes no buscan satisfacer la necesidad de relacionarse con otros, expuesta en la teoría de teoría de la autodeterminación de Deci y Ryan (1985; 2010).

Otro hallazgo interesante fue el surgimiento de dos sub-temas relacionados con la motivación extrínseca: el diseño del curso y la certificación. El formato del diseño del curso afectó diferencialmente la motivación de los participantes, para aquellos con rendimiento alto y medio resaltaron los atributos positivos, y lo contrario para los participantes de rendimiento bajo. Estos hallazgos están respaldados por otros estudios que han explorado el diseño de entornos de aprendizaje en línea (De Freitas, Morgan y Gibson, 2015; Guajardo-Leal et al.,2019; Hew, 2016; Toven-Lindsey, Rhoads, y Lozano, 2015), además de estudios relacionados con la persistencia (Tinto, 2017), aunque no están directamente relacionados con la motivación por aprender. Sobre la certificación, los participantes estuvieron motivados a tomar el curso porque buscaban un reconocimiento con el que pudieran tener ganancias económicas, encontrar empleo, ascender de puesto laboral, recuperar el dinero invertido o aspirar a viajar para laborar en otro país diferente al de su residencia. Los factores exógenos al participante juegan un papel crucial en la motivación por aprender en un xMOOC, su investigación futura es fundamental para comprender los motivos que los llevan a inscribirse en este tipo de cursos.

\subsection{Percepciones sobre persistencia}

Según Bandura (1995; 2006), los individuos con fuerte sentido de autoeficacia tienen un mayor sentido de compromiso e interés en las actividades en las que participan; no obstante, los participantes de rendimiento bajo reportaron fuerte sentido de autoeficacia y voluntad por terminar el curso, sin lograrlo. La autoeficacia y la voluntad no estuvieron asociados a un mejor rendimiento en participantes de xMOOC.

Otro hallazgo en las percepciones sobre persistencia fue encontrar diferencias entre los grupos comparados en la valoración de los materiales y el contenido del curso. Los participantes de rendimiento bajo mostraron cierto descontento e insatisfacción con los contenidos o materiales del curso, lo contrario en los participantes de rendimiento alto y medio. Este hallazgo es congruente con la teoría la persistencia de Tinto (2017) y con el estudio de Tessema, Ready y Yu (2012), en estos se afirma que para persistir los estudiantes deben percibir que el material que se debe aprender es de suficiente calidad y relevancia para así justificar su tiempo y esfuerzo.

También se encontraron diferencias en la forma en la que los participantes de los tres grupos afrontaron la adversidad. En el caso de los participantes con rendimiento bajo el nivel de desafío no fue apropiado para su nivel de habilidad, lo que resultó en aprendizaje superficial y bajos niveles de satisfacción; lo contrario sucedió en los 
participantes de rendimiento medio y alto. Este hallazgo es consistente con la teoría de flujo de Csíkszentmihályi (1996; 2008), en xMOOC un participante puede experimentar relajación al aprender una tarea cuando su nivel de habilidad es muy alto y el desafío de la tarea es muy bajo, y por el contrario, un alumno puede experimentar ansiedad cuando su nivel de habilidad es muy bajo y el desafío de la tarea es muy alto; ninguno de estos estados apoyó la culminación de la meta educativa de los participantes.

\subsection{Conclusiones: implicaciones y recomendaciones}

El reconocer que muchas instituciones de educación media y superior ofrecen cursos vía xMOOC significa que los hallazgos de este estudio pueden ser importantes para desarrolladores de políticas y administradores educativos, desarrolladores y diseñadores de cursos, profesores y estudiantes interesados en tipo de formación. Conocer las percepciones sobre motivación, compromiso y persistencia de los participantes puede ayudar a los programas a desarrollar estrategias para mejorar la eficiencia terminal. Las implicaciones de este estudio incluyen:

1. Las dimensiones de intercambio de conocimiento e interacción sin esfuerzo entre los estudiantes son mucho más fáciles de realizar en ambientes presenciales, esta forma de aprendizaje es casi natural en el aula; no obstante, el apoyo actual que se brinda en xMOOC no es suficiente. Las actividades colaborativas en estos ambientes de aprendizaje deben brindar oportunidades para formar relaciones sociales, de no lograrlo, no serán académicamente valiosas para los participantes. Las herramientas de colaboración que se incorporen en el curso deben facilitar la interacción entre los usuarios para compartir sus ideas, discutir los problemas, proporcionar comentarios y ayudarlos a trabajar para lograr un objetivo de aprendizaje común de manera eficiente. La integración de alguna red social en las actividades colaborativas pudiera ser de beneficio para facilitar la comunicación y el vínculo entre los participantes.

2. Los estudiantes motivados por la relevancia para una carrera futura pueden beneficiarse del contenido de aprendizaje que explora la aplicación del conocimiento en el mundo real. Una proporción significativa de estudiantes está utilizando estos XMOOC para abordar directamente las necesidades de aprendizaje en el lugar de trabajo. Las aplicación del conocimiento debe ser parte fundamental del diseño de ambientes de aprendizaje en xMOOC.

3. Un aprendiz se desarrolla y alcanza su potencial al impulsarse más allá de su capacidad percibida. Encontrarse en un estado óptimo de funcionamiento, ni muy retador que incite a la deserción, ni muy fácil que provoque el aburrimiento, con frecuencia se correlaciona positivamente con el bienestar y el rendimiento. Para alcanzar ese estado óptimo, los cursos deben ofrecer advertencia anticipada a los participantes sobre los siguientes aspectos: las competencias base que deben tener para empatar sus habilidades con el nivel de desafío esperado, el formato del programa, y las expectativas en términos de rendimiento. Cursos que cumplan con estas necesidades pueden atraer candidatos prometedores.

4. El impacto psicológico de las emociones positivas en XMOOC pueden llevar a los participantes a sentirse mejor sobre el aprendizaje. Los estudiantes que tuvieron actitudes positivas hacia lo que se aprende, también sintieron que tenían el control de su propio aprendizaje, lo que conllevó a la aplicación de un mayor esfuerzo. Quizá la integración de estrategias de auto-reconocimiento y retroalimentación sobre las emociones ayude a identificar aquellos estudiantes que pudieran ser 
rescatados antes de desistir, o también facilite la identificación de aquellos participantes que pueden apoyar a otros compañeros.

5. El aprendizaje estratégico es importante para todos los estudiantes, pero es esencial para aquellos que se aventuran en los cursos en línea, masivos y abiertos. El uso de estrategias, tales como elaboración de resúmenes, organización en mapas conceptuales, figuras o gráficos, y planeación y organización de la rutina de estudio, son indispensables para incorporar nueva información en la memoria y para hacer conexiones internas que integren nueva información con el conocimiento previo. Valdría la pena reflexionar sobre cómo se promueve el aprendizaje estratégico en los xMOOC.

6. La certificación es un motivador extrínseco en xMOOC fuertemente relacionado con el nivel de compromiso de los participantes. Sabiendo que no todos los alumnos completan los xMOOC que comienzan, un certificado puede ser un paso valioso para ganar créditos y credenciales sobre el tema y para eventualmente unirse a una comunidad científica de práctica. Si se busca incrementar la tasa de finalización de estos cursos, valdría la pena desarrollar nuevos tipos o niveles de certificación que motiven a los participantes a culminar sus metas educativas.

7. Las formas convencionales de medición de la autoeficacia y voluntad del participante en xMOOC no muestran relación directa con su rendimiento. Es de particular importancia estudiar estos constructos en ambientes en línea, masivos y abiertos en futuros estudios para aspirar una mayor comprensión de su impacto en el comportamiento de los participantes.

El diseño de este estudio presentó algunas limitaciones. Se proporcionó solo una perspectiva sobre las percepciones de motivación, compromiso y persistencia en xMOOC, la de los propios estudiantes, excluyendo otros componentes internos y externos. Asimismo, la muestra es considerada pequeña, aunque cuando se diseña un estudio de caso es común tener pocos participantes. Adicionalmente, no es posible generalizar los hallazgos de este estudio, no obstante, estos brindan luz sobre aspectos de los xMOOC que pudieran ser tomados en cuenta para incrementar las tasas de finalización. A partir de este estudio se puede indicar que para ayudar a que el transitar de los aprendices por este tipo de cursos sea más eficiente, se requiere mayor indagación en el estudio del compromiso, motivación y persistencia de los participantes.

Presentación del artículo: 19 de agosto de 2020 Fecha de aprobación: 12 de abril de 2021

Fecha de publicación: 30 de abril de 2021

Guajardo-Leal, B. E. y Gallardo, K. E. (2021). Compromiso, motivación y persistencia del participante en xMOOC. RED. Revista de Educación a Distancia, 21(66). https://doi.org/10.6018/red.440241

\section{Financiación}

Esta investigación es un resultado del proyecto 266632 "Laboratorio Binacional para la Gestión Inteligente de la Sustentabilidad Energética y la Formación Tecnológica" financiado a través de Fondo CONACYT SENER de Sustentabilidad Energética (S0019201401). 


\section{Referencias}

Anderson, A., Huttenlocher, D., Kleinberg, J., y Leskovec, J. (2014). Engaging with massive online courses. Ponencia presentada en Proceedings of the 23rd international conference on World Wide Web - WWW '14 (pp. 687-698), Seoul, Korea. doi:10.1145/2566486.2568042

Appleton, J. J., Christenson, S. L., y Furlong, M. J. (2008). Student engagement with school: Critical conceptual and methodological issues of the construct. Psychology in the Schools, 45(5), 369-386. doi:10.1002/pits.20303

Bandura, A. (1977). Self-efficacy: Toward a unifying theory of behavioral change. Psychological Review, 84, 191- 215.

Bandura, A. (1995). Self-efficacy. En A. S. R. Manstead y M. Hewstone (Eds.), Blackwell encyclopedia of social psychology (pp. 453-454). Oxford: Blackwell.

Bandura, A. (2006). Going global with social cognitive theory: from prospect to paydirt. En S. I. Donaldson, D. E. Berger, y K. Pezdek (Eds.), The rise of applied psychology: New frontiers and rewarding careers (pp. 53-70). Mahwah, NJ: Erlbaum.

Chemers, M., L. Hu, y Garcia, B. (2001). Academic self-efficacy and first-year college student performance and adjustment. Journal of Educational Psychology, 93(1), 5564

Creswell, J. W. (1998). Qualitative inquiry and research design: Choosing among five traditions. Thousand Oaks, CA: Sage Publications.

Creswell, J. W. (2012). Educational research: Planning, conducting, and evaluating quantitative and qualitative research (4th ed.). Boston, MA: Pearson. doi:10.1017/CBO9781107415324.004

Creswell, J. W. y Guetterman, T. C. (2019). Educational research: Planning, conducting and evaluating cuantitative and qualitative research (6th ed.). New Jersey: Pearson.

Creswell, J. W. y Plano-Clark, V. L. (2018). Designing and conducting mixed methods research (3rd ed.). Thousand Oaks, CA: Sage.

Csíkszentmihályi, M. (1996). Flow and the psychology of discovery and invention. New Yprk: Harper Collins. Chicago

Csíkszentmihályi, M. (2008). Flow: The psychology of optimal experience. New York, NY: Harper Perennial.

Deci, E. L., y Ryan, R. M. (1985). Intrinsic motivation and self-determination in human behavior. New York: Plenum.

Deci, E. L., y Ryan, R. M. (1991). A motivational approach to self-integration in personality. En R. Dienstbier (Ed.), Nebraska symposium on motivation: Perspectives on motivation (Vol. 38, pp. 237-288). Lincoln: University of Nebraska Press. doi:10.1207/s15326985ep2603y4_6

Deci, E. L., y Ryan, R. M. (2000). The "what" and "why" of goal pursuits: Human needs and the self-determination of behavior. Psychological Inquiry, 11(4), 227-268. doi:10.1207/S15327965PLI1104_01

Deci, E. L., y Ryan, R. M. (2010). Self-Determination. New York, NY: John Wiley y Sons, Inc. doi:10.1002/9780470479216.corpsy0834

De Freitas, S. I., Morgan, J., y Gibson, D. (2015). Will MOOC transform learning and teaching in higher education? Engagement and course retention in online learning 
provision. British Journal of Educational Technology, 46(3), 455-471. doi:10.1111/bjet.12268

Fredricks, J. A., Blumenfeld, P. C., y Paris, A. H. (2004). School engagement: Potential of the concept, state of the evidence. Review of Educational Research, 74(1), 59-109. doi: $10.3102 / 00346543074001059$

Ferguson, R., y Clow, D. (2015). Examining engagement: Analysing learner subpopulations in Massive Open Online Courses (MOOC). Ponencia presentada en Proceedings of the Fifth International Conference on Learning Analytics And Knowledge - LAK '15 (pp. 51-58). Poughkeepsie, NY, EE.UU. doi: $10.1145 / 2723576.2723606$

Guajardo-Leal, B. E., Navarro-Corona, C., y Valenzuela, J. R. (2019). Systematic mapping study of academic engagement in MOOC, International Review of Research in Open and Distributed Learning, 20(2), 114-139.

Guajardo-Leal, B. E., Valenzuela, J. R. y Scott, J. (2019). Student engagement as predictor of xMOOC completion: An analysis from five courses on energy sustainability, Online Learning Journal. 23(2), 105-123. Recuperado de: http://hdl.handle.net/11285/632315

Halawa, S., Greene, D., y Mitchell, J. (2014). Dropout prediction in MOOC using learner activity features. eLearning Papers, 37(March), 1-10.

Hew, K. F. (2016). Promoting engagement in online courses: What strategies can we learn from three highly rated MOOC. British Journal of Educational Technology, 47(2), 320-341. doi:10.1111/bjet.12235

Joksimović, S., Poquet, O., Kovanović, V., Dowell, N., Mills, C., Gašević, D., Dawson, S., Graesser, A.C., y Brooks, C. (2018). How do we model learning at scale? A systematic review of research on MOOC. Review of Educational Research, 88(1), 4386. doi:10.3102/0034654317740335

Jordan, K. (2014). Initial trends in enrolment and completion of massive open online courses Massive Open Online Courses. International Review of Research in Open and Distance Learning, 15(1), 133-160. doi:10.19173/irrodl.v15i1.1651

Kizilcec, R. F., Pérez-Sanagustín, M., y Maldonado, J. J. (2016). Self-regulated learning strategies predict learner behavior and goal attainment in Massive Open Online Courses. Computers and Education, 1-15.

Krause, K. (2005). Understanding and promoting student engagement in university learning communities. Ponencia presentada en Engaged, Inert or Otherwise Occupied?: Deconstructing the 21st Century Undergraduate Student, James Cook University Symposium 'Sharing Scholarship in Learning and Teaching: Engaging Students'. Queensland, Australia.

Martin, N. I., Kelly, N., y Terry, P. C. (2018). A framework for self-determination in massive open online courses: Design for autonomy, competence, and relatedness. Australasian Journal of Educational Technology, 34(2), 35-55. doi:10.14742/ajet.3722

Mosher, R., y MacGowan, B. (1985). Assessing Student Engagement in Secondary Schools: Alternative Conceptions, Strategies of Assessing, and Instruments. University of Wisconsin, Research and Development Center.

Pintrich, P. R., y De Groot, E. (1990). Motivated and self-regulated learning components of academic performance. Journal of Educational Psychology, 82, 3340.

Peterson, C. y Seligman, M. (2004). Character strengths and virtues: A handbook and classification. Oxford: Oxford University Press. 
Redmond, P., Heffernan, A., Abawi, L., Brown, A., y Henderson, R. (2018). An online engagement framework for higher education. Online Learning, 22(1), 183-204. doi:10.24059/olj.v22i1.1175

Reeve, J. (2012). A self-determination theory perspective on student engagement. En S. L. Christenson, C. Wylie, y A. L. Reschly (Eds.), Handbook of Research on Student Engagement (pp. 149-172). doi:10.1007/978-1-4614-2018-7

Reschly, A. L., y Christenson, S. L. (2012). Jingle, jangle, and conceptual haziness: Evolution and future directions of the engagement construct. En S. L. Christenson, A. L. Reschly, y C. Whlie (Eds.), Handbook of Research on Student Engagement (pp. 320). New York, NY: Springer.

Stake, R. E. (1995). The art of case study research. Thousand Oaks, CA: Sage Publications.

Tessema, M., Ready, K., y Yu, W. (2012). Factors affecting college students' satisfaction with major curriculum: Evidence from nine years of data. International Journal of Humanities and Social Science, 2(2), 34-34. Recuperado de: http://www.ijhssnet.com/

Tinto, V. (2017). Reflections on student persistence. Student Success, 8(2), 1. doi:10.5204/ssj.v8i2.376

Toven-Lindsey, B., Rhoads, R. A., y Lozano, J. B. (2015). Virtually unlimited classrooms: Pedagogical practices in massive open online courses. Internet and Higher Education, 24, 1-12. doi:10.1016/j.iheduc.2014.07.001

Wen, M., Yang, D., y Rosé, C. P. (2014). Linguistic reflections of student engagement in massive open online courses. Ponencia presentada en the Eighth International Conference on Weblogs and Social Media. Palo Alto, California.

White, R. (1959). Motivation reconsidered: The concept of competence. Psychological Review, 66, 279-333.

Xiong, Y., Li, H., Kornhaber, M. L., Suen, H. K., Pursel, B., y Goins, D. D. (2015). Examining the relations among student motivation, engagement, and retention in a MOOC: A structural equation modeling approach. Global Education Review, 2(3), 23-33.

Yin, R. (2003). Case study research: Design and methods (3rd Ed.). Thousand Oaks, CA: Sage Publications.

Yuan, L., y Powell, S. (2013). MOOCs and open education: Implications for higher education. Bolton: CETIS. Recuperado de: http://publications.cetis.ac.uk/2013/667

Zimmerman, B. J. (2008). Investigating self-regulation and motivation: historical background, methodological developments, and future prospects. American Educational Research Journal, 45(1), 166-183. doi:10.3102/0002831207312909 